\title{
The AKARI mid-infrared all-sky survey: development of a new inter-planetary dust map and the world's first all-sky PAH map
}

\author{
Daisuke Ishihara, Hidehiro Kaneda, Toru Kondo, Tomoya Amatsutsu, \\ Keiichiro Nakamichi, Mitsuyoshi Yamagishi, Shinki Oyabu \\ Department of physics, Graduate school of Science, Nagoya University \\ Furo-cho, Chikusa-ku, Nagoya, Aichi, Japan \\ E-mail: ishihara@u.phys.nagoya-u.ac.jp
}

\section{Takafumi Ootsubo}

Department of physics, Graduate school of Science, Tohoku University

Aoba-ku, Sendai, Miyagi, Japan

\section{Takashi Onaka}

Department of astronomy, Graduate school of Science, University of Tokyo 3-1-1, Hongo, Tokyo, Japan

We are constructing accurately calibrated $9 \mu \mathrm{m}$ and $18 \mu \mathrm{m}$ all-sky diffuse maps from the AKARI mid-infrared all-sky survey data. These maps are heavily affected by the foreground emission of the zodiacal light, which has an intensity peak at around these wavelengths. We carefully separate the zodiacal emission component from the maps using Kelsall's model. Through improvement of the parameters in the zodiacal light emission model, we obtained new insight on the structure and composition of the interplanetary dust in our solar system.

The zodiacal light removed AKARI $9 \mu \mathrm{m}$ map is the world's first all-sky PAH map, that traces the emission features of Galactic polycyclic aromatic hydrocarbons (PAHs) at wavelengths of $6.2,7.7,8.6$, and $11.3 \mu \mathrm{m}$. On a global scale, PAHs show good spatial correlation with tracers of general ISM such as CO, HI, and far-IR dust emissions. On a local scale, we recognize the variation of physical state and compositions of hydrocarbons reflecting the variation of the local physical environment. This PAH map will be effectively used in diagnoses of various interstellar phenomena.

The Life Cycle of Dust in the Universe: Observations, Theory, and Laboratory Experiments - LCDU 2013, 18-12 November, 2013

Taipei, Taiwan 


\section{Introduction}

AKARI has made all-sky surveys in $9,18,65,90,140$, and $160 \mu \mathrm{m}$ bands. We are constructing diffuse maps from the mid-infrared $(9$ and $18 \mu \mathrm{m})$ bands data. These maps have higher spatial resolution ( $\sim$ ") and higher sensitivity [1] than the previous IRAS 12 and $25 \mu \mathrm{m}$ all-sky maps. Moreover, the $9 \mu \mathrm{m}$ band efficiently traces the emission features of the interstellar polycyclic aromatic hydrocarbons (PAHs). PAHs are small organic matters in the interstellar space. They typically consist of a few tens of carbon atoms and have sizes of about a few tens Angstrom. They are ubiquitous in the interstellar media (ISM) in our Galaxy and nearby galaxies [2] and seem to be well mixed with neutral medium traced by HI gas, molecular gas, and dust grains emitting at far-infrared wavelengths [3]. They show infrared features at around 6.6, 7.2, 8.6, and $11.3 \mu \mathrm{m}$ as shown in Fig. 1a. Among the mid-infrared maps opened to the public, only the AKARI $9 \mu \mathrm{m}$ map shows structures reflecting PAH emission rather than hot dust emission (Fig. 1b).
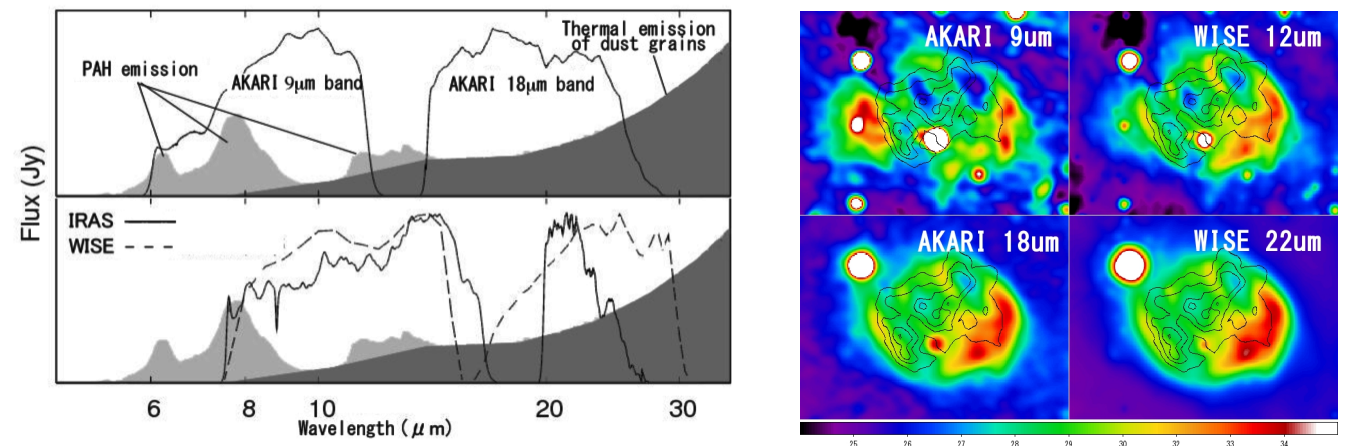

Figure 1: (a) A typical spectrum of a HII region [4] showing PAH features. Filter response curves of mid-IR bands of AKARI, IRAS, and WISE are overlaid. (b) Examples of diffuse maps around W44B observed with AKARI 9 and $18 \mu \mathrm{m}$ bands and WISE 12 and $22 \mu \mathrm{m}$ bands.

\section{Subtraction of inter-planetary dust emission}

Fig. 2a shows the AKARI $9 \mu \mathrm{m}$ all-sky raw data in Galactic coordinates. We can see a dominant component along the ecliptic plane in addition to the Galactic plane emission. It is the zodiacal light, corresponding to thermal emission from interplanetary dust in our Solar system. For studies of the Galactic PAH emission, we have to subtract the zodiacal light emission accurately from the map. Fig $2 \mathrm{~b}$ shows the $9 \mu \mathrm{m}$ Galactic emission map in which the zodiacal light is removed using the standard model of Kelsall [5]. The residual component is at the $\sim 6 \%$ level of the raw data intensity. It shows good similarity with widely used IRAS $12 \mu \mathrm{m}$ map (Fig. 2c). But the residual level is still large compared to the faint Galactic PAH emission. 

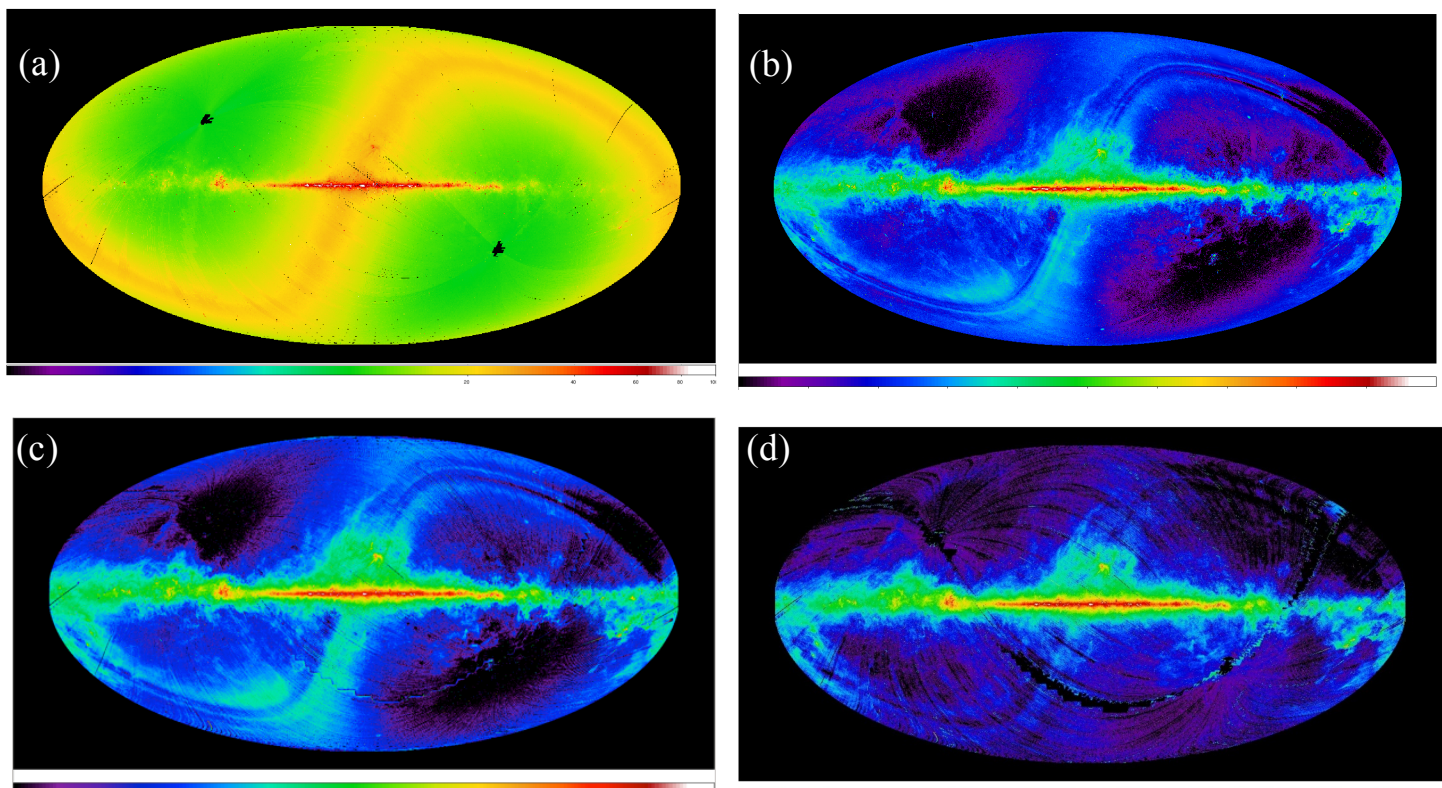

Figure 2: (a) AKARI $9 \mu \mathrm{m}$ all-sky survey raw data in Galactic coordinates. It is dominated by the Galactic plane emission and the thermal emission of IPDs around the ecliptic plane. (b) Zodiacal light is subtracted from (b) using Kelsall's model. (c) Zodiacal emission subtracted IRAS 12 um map [7]. (d) Zodiacal light is subtracted from (b) using our modified Kelsall's model.

Then, we optimized the parameters (e.g. emissivity modification factors, geometrical parameters of individual cloud components) in Kelsall's model to reduce the residual component level. In previous studies [5], only the seasonal fluctuation of the zodiacal light is fitted with the model to exclude the static components such as Galactic emission. In this work, we make simultaneous fitting of each seasonal chunk of raw data with the model after masking the Galactic component. By this modification, we can explain the static component of the zodiacal light in addition to the time-varying component by the model. As a result, some parameters are significantly changed. Through improvement of parameters in the zodiacal light emission model, we obtained the revised information on the structure and composition of the IPD in our solar system. Details are summarized in [6].

\section{Galactic PAH emission}

We subtracted the zodiacal light component from the AKARI $9 \mu \mathrm{m}$ all-sky map using the modified Kelsall's model. Fig. 2d shows the result. The residual component level is successfully reduced to be below $\sim 2 \%$ of the original raw data level.

We can now use this map to study various interstellar phenomena linked to the faint extended Galactic PAH emission. For example, it turns out that there are local regions where the PAH emission is relatively weak compared with the far-IR dust emission [3]. These regions correspond to the foot points of the Galactic molecular loop, a buoyant rise of magnetic loops 
due to magnetic flotation driven by the Parker instability. The relatively weak local PAH/dust intensity ratio suggests shock destruction of PAHs in these regions.

Based on the all-sky PAH map, a large-scale excess PAH emission around the Galactic center is revealed [7]. This component is not associated with nearby clouds and seems to be associated with the Galactic center region. It is spatially correlated with Fermi bubbles, a gamma-ray emission related to the past outflows from the Galactic center. If the overabundance of PAHs in this region is the result of shuttering of carbonaceous grains in past outflows, it indicates the past activities in the Galactic center.

\section{Summary}

The AKARI $9 \mu \mathrm{m}$ map is a unique resource as a world-first all-sky PAH tracer. In order to create accurate PAH emission maps, we revise the zodiacal light model and improve the accuracy of the zodiacal light subtraction. Using our zodiacal light subtracted all-sky PAH map, we can diagnose various interstellar phenomena because the local variance of $\mathrm{PAH} / \mathrm{dust}$ intensity ratio reflects physical properties of the region.

\section{Acknowledgements}

This research is based on observations with AKARI, a JAXA project with the participation of ESA. This work is supported by JSPS KAKENHI Grant Number 24740122.

\section{References}

[1] Ishihara, D., et al., The AKARI/IRC mid-infrared all-sky survey, A\&A, 514, A1 (2010)

[2] Onaka, T., et al., Detection of the Mid-Infrared Unidentified Bands in the Diffuse Galactic Emission by IRTS, PASJ, 48, 59 (1996)

[3] Kaneda, H., et al., Processing of Polycyclic Aromatic Hydrocarbons in Molecular-Loop Regions near the Galactic Center Revealed by AKARI, PASJ, 64, 25 (2012)

[4] Sloan, G. C., et al., A uniform database of 2.4-45.4 micron spectra from the ISO Short Wavelength Spectrometer, ApJS, 147, 379 (2003)

[5] Kelsall, T., et al., The COBE Diffuse Infrared Background Experiment Search for the Cosmic Infrared Background. II. Model of the Interplanetary Dust Cloud, ApJ, 508, 44 (1998)

[6] Kondo, T., et al., Large-scale extraplanar PAH emission in the Galactic center region found by AKARI mid-infrared all-sky survey, in this volume PoS (LCDU 2013) 033

[7] Miville-Deschênes, M.-A., and Lagache, G., IRIS : A new generation of IRAS maps, ApJS, 157, 302 (2005) 